\title{
DIFFERENCES BETWEEN ARTERIAL, VENOUS AND INTRAOSSEOUS BLOOD SAMPLES IN POINT-OF-CARE ANALYSES DURING EXPERIMENTAL CPR
}

\section{Milla Jousi ${ }^{1,2,3}$, Markus Skrifvars ${ }^{2,3}$, Annika Nelskylä ${ }^{2,3}$, Giuseppe Ristagno $^{4}$, Alexey Schramko ${ }^{3}$, Jouni Nurmi ${ }^{2}$}

\author{
${ }^{1}$ FinnHEMS Research and Development Unit, Finland \\ ${ }^{2}$ Emergency Medicine, University of Helsinki and Department of Emergency Medicine and Services, Helsinki University Hospital, Finland \\ ${ }^{3}$ Department of Anaesthesiology, Intensive Care and Pain Medicine, Helsinki University and Helsinki University Hospital, Finland \\ ${ }^{4}$ Istituto di Ricerche Farmacologiche "Mario Negri", Milano, Italy
}

\section{Purpose of the study}

Resuscitation guidelines recommend assessing and correcting reversible causes of cardiac arrest (CA) during cardiopulmonary resuscitation (CPR) i.e. by using the $4 \mathrm{H}$ 's and $4 \mathrm{~T}$ 's rule.

Point-of-care (POC) laboratory analyses could probably supplement clinical information in screening pre-arrest pathologies, i.e. electrolyte disorders, acid-base balance disturbances and abnormal haemoglobin levels.

Because arterial or venous blood samples may be difficult to obtain during CPR, we aimed to study how POC samples from intraosseous (IO) space during resuscitation resemble the prearrest arterial values.
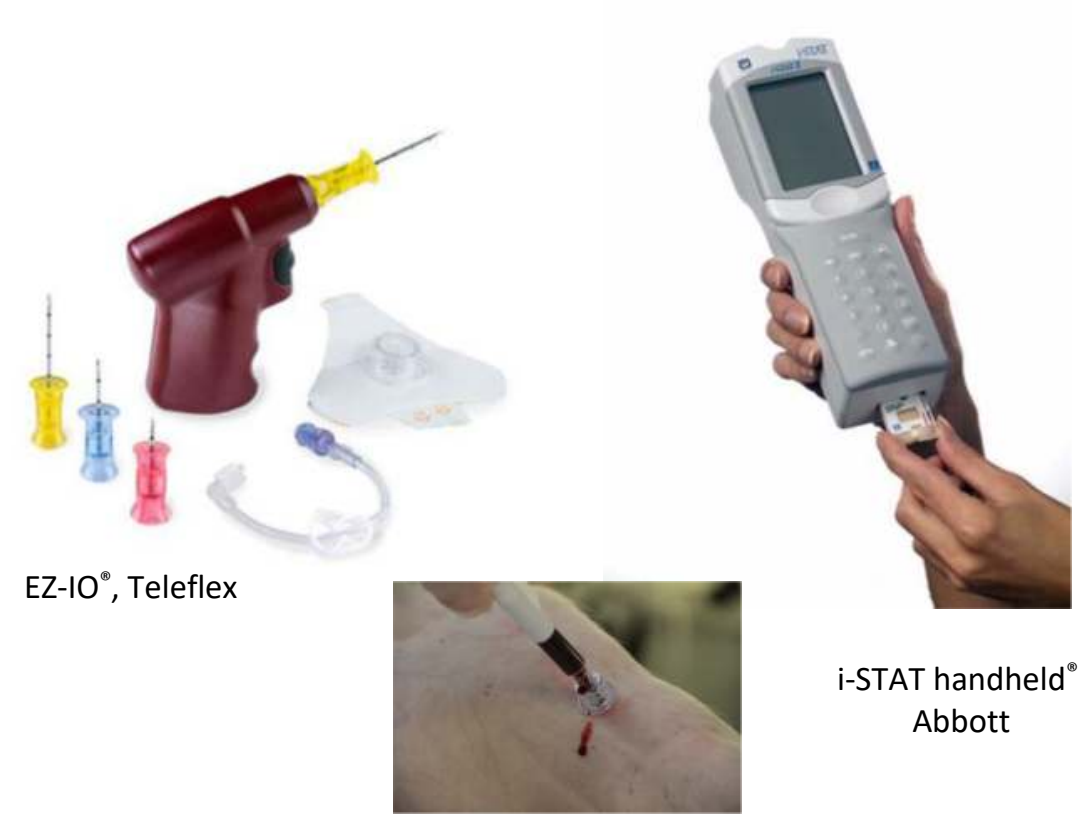

\section{Materials and methods}

TIMELINE OF THE EXPERIMENT

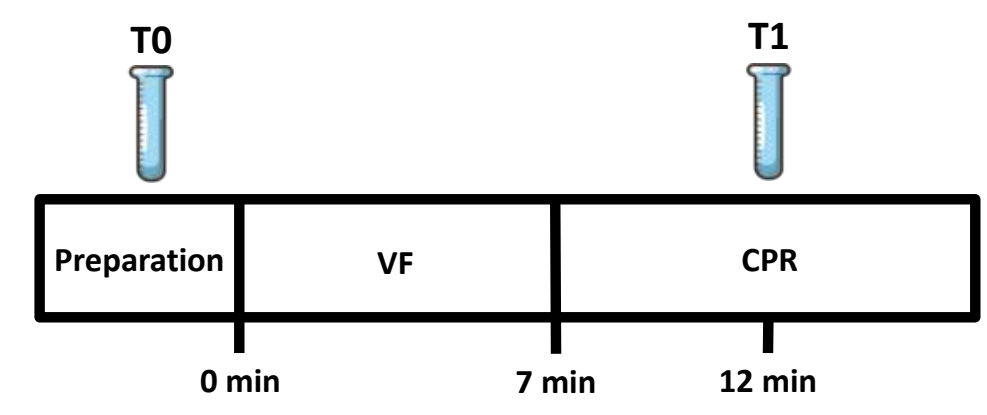

To
T1
VPR
CPR

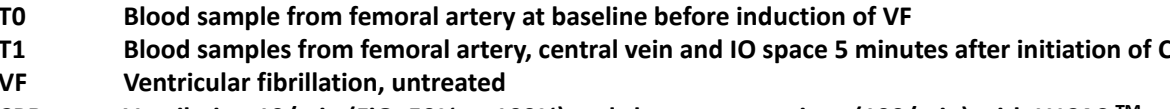

VF
CPR Ventricular fibrillation, untreated
Ventilation $10 /$ min (Fii $20 \%$ or $100 \%$ ) and chest compressions (100/min) with LUCAS TM

We used an experimental resuscitation model with 23 anesthetised pigs. The Finnish National Animal Experiment Board (ESAVI/1077/04.10.07/2016) approved the study plan.

We took baseline blood samples from artery before electrically inducing VF and compared the baseline arterial samples with arterial, venous and IOsamples taken after 5 minutes of CPR, which was started after 7 minutes of untreated CA. We used iSTAT $^{\oplus}$ POC device for analyses and Friedman's two-way ANOVA and its post hoc pairwise comparisons with Bonferroni correction for the statistical analyses.

\section{Results}

Electrolyte and acid-base values from all sampling sites during resuscitation differ from the pre-arrest values. Figures below display the difference between arterial, IO-, and venous values during CPR compared to the pre-arrest arterial values.

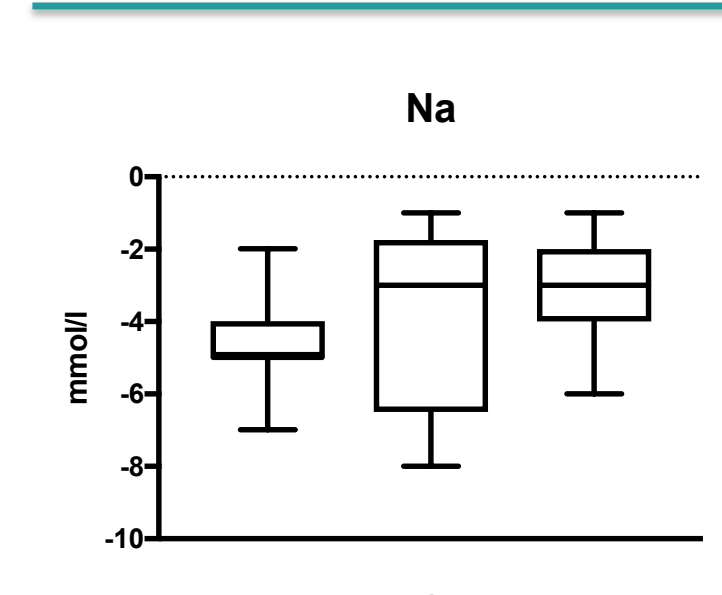

ART IO VEIN

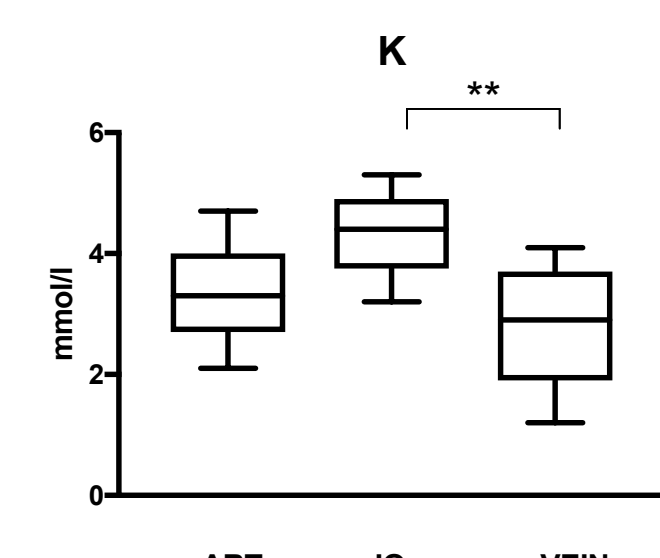

ART IO VEIN

\section{Conclusions}

Venous, arterial and IO POC samples during CPR resemble poorly the prearrest values.

POC analysis of 10 samples is not inferior to analyses of arterial or venous blood.

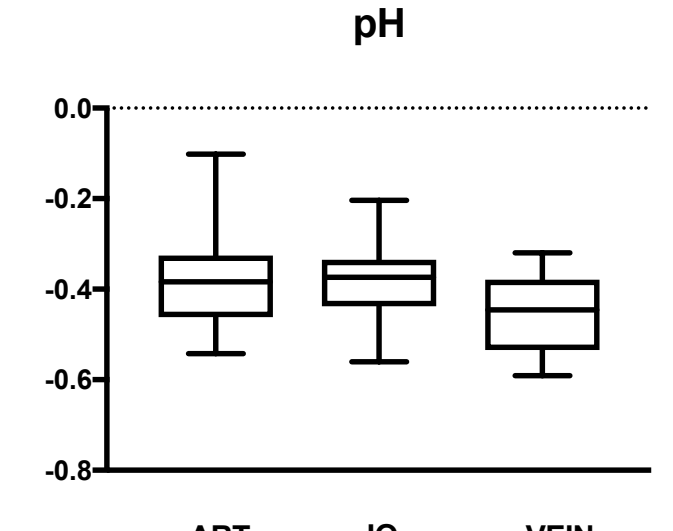

ART IO VEIN

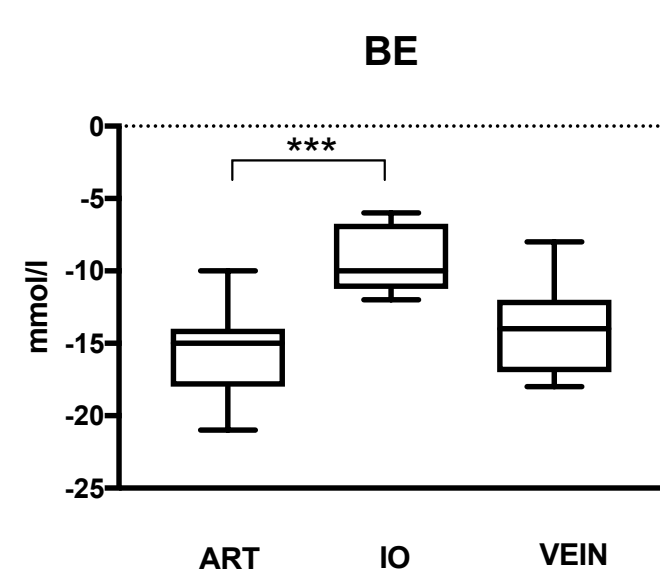

ART Difference ARTERIAL CPR value (T1) - ARTERIAL BASELINE value (TO) $\begin{array}{ll}\text { IO } & \text { Difference IO CPR value (T1) - ARTERIAL BASELINE value (TO) } \\ \text { VEIN } & \text { Difference VENOUS CPR value (T1) - ARTERIAL BASELINE value (TO) }\end{array}$

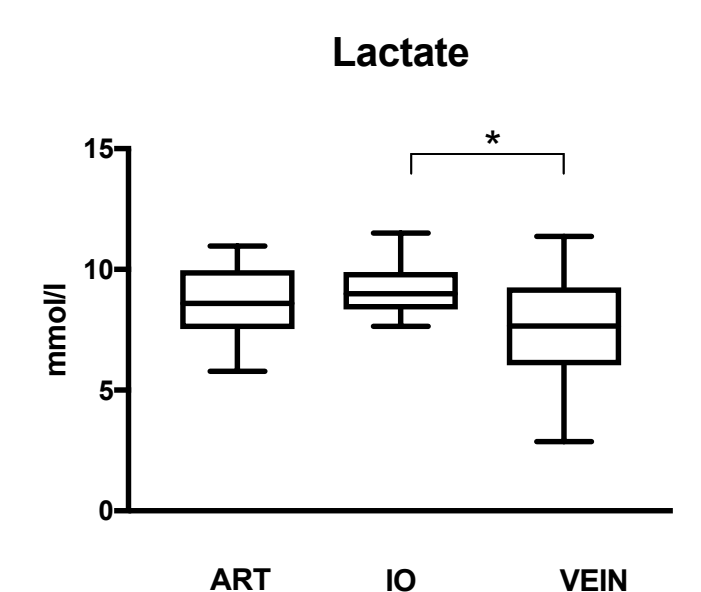

ART IO VEIN

$\mathrm{HCO}_{3}$

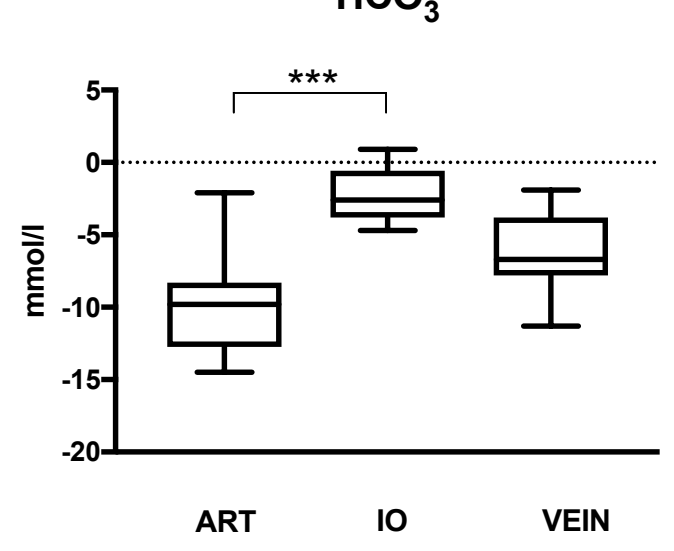

$p<0.05$ $\begin{array}{cc}* * & \mathrm{p}<0.01 \\ * * * & \mathrm{p}<0.001\end{array}$ 